\title{
A study on relationship between elderly sarcopenia and inflammatory factors IL-6 and TNF-a
}

\author{
Ai-Lin Bian*, Hui-Ying Hu, Yu-Dong Rong, Jian Wang, Jun-Xiong Wang and Xin-Zi Zhou
}

\begin{abstract}
Background: This report aims to study the relationship between sarcopenia of elderly in community and inflammatory factors IL-6 and TNF-a.

Methods: A total of 441 elders who undertook physical examinations were included into this study. The age of these subjects were $>60$, in which 235 subjects were male and 206 subjects were female. According to the diagnostic standards of sarcopenia set by EWGSOP and AWGS, these subjects were divided into two groups: sarcopenia, and non-sarcopenia groups. The living habits, disease status, biochemical indexes, and levels of IL-6 and TNF-a of these subjects were investigated.

Results: The morbidity rate of sarcopenia was $17.02 \%$ in male subjects and $18.9 \%$ in female subjects. In elderly subjects $>80$ years old, morbidity rate was $25.3 \%$ in male subjects and $35.1 \%$ in female subjects. The history of smoking in patients with sarcopenia was long, and their regular exercise history was short $(P<0.01)$. Furthermore, differences in handgrip strength $(\mathrm{HG})$, fat-free mass (FFM), bone mineral content (BMC), plasma albumin (ALB) and serum creatinine $(\mathrm{Cr})$, and body fat content (FAT) in patients between the sarcopenia and non-sarcopenia groups were statistically significant $(P<0.05)$. Moreover, differences in IL-6 and TNF-a levels between these two groups were statistically significant $(P<0.05)$. In addition, BMI was positively correlated to TNF-a levels, and ALB was negatively correlated to IL-6; while BMI and VFA were positively correlated to TNF-a levels, and SMM, HDL-C, Hb, HG were negatively correlated to IL-6 level $(P<0.05)$. Multiple linear regression analysis suggested plasma ALB and BMI were the independent risk factors of TNF-a, while VFA was the independent risk factor of IL-6.
\end{abstract}

Conclusions: The onset of sarcopenia was associated with poor exercise habits, disease history, and nutritional status. The emergence of sarcopenia was accompanied by increased levels of inflammation factors TNF-a and IL-6. Plasma albumin, BMI, and VFA were inflammatory factor predictors of TNF and IL-6.

Keywords: Elders, Skeletal muscle reduction, IL-6, TNF-a

\section{Background}

With the rapid aging of the population, chronic diseases in the elderly have become a major social problem worldwide. Among the elderly diseases, in addition to diabetes, cardio-cerebrovascular diseases, tumors and other frequently occurring diseases, the health problems caused by changes in the body compositions of the elderly are

*Correspondence: ailinbiandoc@163.com Department of Geratology, Tianjin First Central Hospital, No. 24 of Fukang Road, Nankai District, Tianjin 300192, China easy to be neglected. As the body aged, the composition of the body also changed such as the increase in adipose tissues and reduction in muscle tissues. Foreign studies have shown that age-induced sarcopenia has become an important health problem that affects the quality of life of the elderly. In 1989, Ronsenberg first proposed the definition of sarcopenia, that is, a progressive decline in skeletal muscle mass and strength. Its negative effects on the elderly could be observed in many aspects: increased risk of falling down and fracture, increased re-admission rates, and increased mortality [1]. Studies have revealed 
that age-related body composition changes, as well as chronic inflammation and proinflammatory factors produced by adipose tissues, are closely related to sarcopenia [2-4]; and this inflammatory reaction may play a key role in the occurrence and development processes of sarcopenia. The purpose of this study is to investigate the relationship between changes in body composition and the incidence of sarcopenia vs. inflammatory factors interleukin-6 (IL-6) and tumor necrosis factor- $\alpha$ (TNF- $\alpha$ ).

\section{Methods}

\section{Research subjects}

This cross-sectional study selected a total of 441 elderly subjects $>60$ years old, who underwent physical examinations in Tianjin First Central Hospital between May 2015 and September 2015. Among these subjects, 235 subjects were male (mean age: $75.31 \pm 8.29$ years old) and 206 subjects were female (mean age $75.30 \pm 3.46$ years old). Furthermore, among these subjects, 161 subjects were $\geq 80$ years old, which included 87 male subjects and 74 female subjects.

\section{Inclusion criteria}

Subjects $>60$ years old, who could walk by themselves or stand for 5 min with other auxiliaries, and had no history of mental illness were enrolled into this study.

\section{Exclusion criteria}

(1) Subjects with tumor or severe weakness. (2) Subjects with diseases that seriously affect their health such as serious arrhythmia, heart failure, infection, edema, or massive ascites. (3) Subjects with endocrine or metabolic diseases that failed to be well-controlled such as type- 2 diabetes and thyroid gland disease. (4) Subjects who were taking anti-inflammation drugs. (5) Autoimmune diseases.

\section{Diagnostic criteria and grouping of sarcopenia subjects Diagnostic criteria}

The diagnostic criteria were based on the diagnostic criteria of sarcopenia established by the European Working Group on Sarcopenia in Older People (EWGSOP) in 2010 and the Asian Working Group for Sarcopenia (AWGS) in 2015, which include the decrease in muscle mass, muscle strength, and/or body function. Muscle mass was evaluated using the appendicular skeletal muscle index (ASMI), which is equal to the appendicular skeletal muscle mass (ASM)/the square of the height $\left(\mathrm{m}^{2}\right)$. The standard evaluation is that the value is less than two mean standard deviations (SD) of the mean value of the youth with the same race and gender, combined with the recommendations of AWGS and EWGSOP. A value $<7.0 \mathrm{~kg} / \mathrm{m}^{2}$ for male subjects and a value $<5.7 \mathrm{~kg} /$ $\mathrm{m}^{2}$ in female subjects were adopted as ASMI cut-off values, respectively. Handgrip strength (HG) is an indicator of muscle strength. A HG strength value $<26 \mathrm{~kg}$ for male subjects and a HG strength value $<18 \mathrm{~kg}$ for female subjects were set as cut-off values.

\section{Grouping method}

All subjects were divided into two groups, according to ASMI and HG strength:

(1) Sarcopenia group: In male subjects, ASMI was $<7.0 \mathrm{~kg} / \mathrm{m}^{2}$ and HG strength was $<26 \mathrm{~kg}$. In female subjects, ASMI was $<5.7 \mathrm{~kg} / \mathrm{m}^{2}$ and $\mathrm{HG}$ strength was $<18 \mathrm{~kg}$.

(2) Non-sarcopenia group: In male subjects, ASMI was $\geq 7.0 \mathrm{~kg} / \mathrm{m}^{2}$, and in female subjects, ASMI was $\geq 5.7 \mathrm{~kg} / \mathrm{m}^{2}$.

\section{Detection index}

\section{Body composition analysis}

Bioelectrical impedance analysis (BIA) was employed to measure the composition of the human body using an InBody S10 body composition analyzer (Biospace Co., Ltd., South Korea). Measurement indexes included the following: intracellular water (ICW), extracellular water (ECW), appendicular skeletal lean mass (SLM), body fat content (FAT), fat-free body mass (FFM), bony mineral content (BMC), protein content (pro), body cell mass $(\mathrm{BCM})$, body mass index (BMI), arm circumference (AC), arm muscle circumference (AMC), and waist circumference (WC).

\section{Body measurements}

(1) Body weight $(\mathrm{kg})$, height $(\mathrm{CM})$, and blood pressure (mmHg) measurements;

(2) HG strength determination: HG strength was determined using a hand-muscle developer (WCSII, Beijing). Each hand was tested twice, the maximum value was used, and these values were corrected to $0.1 \mathrm{~kg}$.

\section{Determination of laboratory indicators}

Clinical inspection indexes Subjects fasted for $8 \mathrm{~h}$. In the morning, $5 \mathrm{ml}$ of fasted elbow venous blood was drawn into a vacuum mining vessel, placed in static for $30 \mathrm{~min}$, and the serum was isolated at $4{ }^{\circ} \mathrm{C}$. Indicators included routine blood, including hemoglobin $(\mathrm{Hb})$, and lymphocyte (Lym) and blood biochemistry, including total cholesterol (TC), triacylglycerol (TG), blood glucose (Glu), serum albumin (ALB), and creatinine (Cr). The detection of the above indicators was completed on the same day. At the same time, another $400 \mu \mathrm{l}$ of serum was packed into two Eppendorf tubes, placed in the refrigerator at $-70{ }^{\circ} \mathrm{C}$, 
and were prepared for the batch determination of IL-6 and TNF- $\alpha$.

Detection of inflammatory factors

(1) Detection of TNF- $\alpha$ : TNF- $\alpha$ was determined by solid-phase sandwich enzyme-linked immunosorbent assay. The kit was produced by RapidBio (RB, USA).

(2) Detection of IL-6: IL-6 was determined by the competitive inhibition of enzyme-linked immunosorbent assay. The kit was produced by RapidBio (RB, USA).

\section{Social demographic factors and way of life}

Social demographic factors were obtained through questionnaires, which included marital status, living environment, smoking history, drinking history, and daily exercise. Previous medical history included hypertension, coronary heart disease, diabetes, and cerebral infarction history.

(1) Definition standards of regular exercise: Exercise $>3$ times a week for $>30$ min each time, which persisted for at least 6 months. All these above were considered as regular exercise.

(2) Definition standard of smoking: According to the definition of active smokers and passive smokers established by the World Health Organization (WHO), that is, people who have smoked for six consecutive months or six cumulative months, or smoked more than 6 months in a lifetime were defined as cigarette smokers. These subjects were divided into two categories: smokers and nonsmokers.

(3) Definition standard of drinking: These subjects were divided into two categories, according to alcohol drinking habits: drinkers and non-drinkers.

\section{Statistical methods}

Data were analyzed using Windows SPSS 19.0 statistical software package. Normal distributed data were expressed as mean \pm standard deviation $(x \pm \mathrm{SD})$, and were analyzed by $t$ test. Count data were analyzed by $X^{2}$ test. Correlations were analyzed by Spearman's rank correlation method. Linear regression and multiple linear regression equations were used for multivariate analysis. $P<0.05$ was considered statistically significant.

\section{Results}

(1) Among these 441 subjects, 79 subjects had sarcopenia including 40 male subjects (17.02\%) and 39 female subjects (18.9\%). Furthermore, among these 79 subjects, 48 subjects were $>80$ years old including 22 male subjects (25.3\%) and 26 female subjects
(35.1\%). Comparisons on the general information, life habits, disease history, body composition, and biochemical indicators of patients between these two groups are listed in Table 1. In life habits, patients with sarcopenia had a long history of smoking and less regular exercise, compared with non-sarcopenia patients; and there was a significant difference between these two groups $(P<0.01)$. For status of illness, more patients suffered from coronary heart disease in the sarcopenia group, and the difference was statistically significant between these two groups $(P<0.05)$. For body composition, differences in height, weight, HG strength, ICW, ECW, pro, FFM, and BMC in male subjects and height, HG strength, ICW, ECW, pro, FAT, FFM, BMC, and VFA in female subjects between the sarcopenia and control groups were statistically significant $(P<0.01)$. For clinical biochemical indexes, differences in levels of DBP, ALB, and $\mathrm{Cr}$ in male subjects and levels of DBP, ALB, Cr, and $\mathrm{Hb}$ in female subjects between the sarcopenia and control groups were statistically significant $(P<0.05)$.

"Height, the height of a person; weight, body weight; HG, handgrip or handgrip strength; ICW, intracellular water; ECW, extracellular water; pro, protein; FAT, fat content of the body; FFM, fat-free body weight; BMI, body mass index; $\mathrm{BMC}$, bone mineral content; AC, upper arm circumference; AMC, arm muscle circumference; WC, waist circumference; VFA, visceral fat area; SBP, systolic blood pressure; DBP, diastolic blood pressure; ALB, plasma albumin; Cr, serum creatinine; Glu, blood glucose; TC, total cholesterol; TG, triglyceride; Lym, blood lymphocyte count; HB, hemoglobin; Hyper, hypertension; Cardiac, coronary heart disease; $\mathrm{NC}$, cerebral vascular disease; DM, diabetes mellitus; Smoke, smoking history; Drink, drinking history; Sport, exercise history.

(2) Correlation analysis of body composition and sarcopenia is shown in Table 2. ICW, ECW, Pro, FFM, $\mathrm{BMC}, \mathrm{AC}$, and $\mathrm{AMC}$ body compositions were correlated to sarcopenia, and these correlations were significantly positive $(P<0.01)$. However, FAT had a significant negative correlation with sarcopenia $(P<0.05)$.

(3) Correlation analysis of sarcopenia and blood biochemical indexes is shown in Table 3. There was a significant positive correlation between sarcopenia and the indexes of DBP, ALB, and $\mathrm{Cr}(P>0.05)$.

(4) Correlation analysis of inflammatory factor and other indicators: The levels of inflammatory factors IL- 6 and TNF- $\alpha$ in the sarcopenia and control groups are shown in Table 4. IL-6 and TNF- $\alpha$ levels 
Table 1 A comparison of clinical data between muscle decrease disease group and non-muscle decrease disease group

\begin{tabular}{|c|c|c|c|c|}
\hline \multirow[t]{2}{*}{ Observation index } & \multicolumn{2}{|c|}{ Muscle decrease disease $(n=79)$} & \multicolumn{2}{|c|}{ Muscle decrease disease $(n=362)$} \\
\hline & $\operatorname{Man}(n=40)$ & Female $(n=39)$ & $\operatorname{Man}(n=195)$ & Female $(n=167)$ \\
\hline ASMI & $7.69 \pm 1.02$ & $5.27 \pm 0.81$ & $8.25 \pm 1.34$ & $7.07 \pm 1.27$ \\
\hline Age & $70.88 \pm 7.23$ & $79.78 \pm 4.32$ & $73.54 \pm 7.81$ & $80.82 \pm 8.34$ \\
\hline Height & $166.28 \pm 5.31^{*}$ & $153.72 \pm 5.30^{*}$ & $171.82 \pm 8.10$ & $160.95 \pm 5.84$ \\
\hline Weight & $67.43 \pm 10.73^{*}$ & $59.34 \pm 17.11$ & $73.35 \pm 11.34$ & $61.90 \pm 9.15$ \\
\hline$H G$ & $30.91 \pm 7.62^{*}$ & $19.65 \pm 6.96^{*}$ & $33.82 \pm 8.14$ & $21.17 \pm 5.52$ \\
\hline ICW & $23.18 \pm 4.03^{*}$ & $15.67 \pm 2.64^{*}$ & $25.89 \pm 4.50$ & $19.46 \pm 3.00$ \\
\hline ECW & $14.58 \pm 2.34^{*}$ & $10.58 \pm 1.69^{*}$ & $16.51 \pm 2.38$ & $12.36 \pm 1.55$ \\
\hline Pro & $10.01 \pm 1.74^{*}$ & $6.77 \pm 1.14^{*}$ & $11.19 \pm 1.95$ & $8.41 \pm 1.29$ \\
\hline FAT & $16.33 \pm 8.91$ & $23.76 \pm 13.32^{*}$ & $16.26 \pm 7.76$ & $18.70 \pm 7.78$ \\
\hline FFM & $51.10 \pm 8.19^{*}$ & $35.59 \pm 5.63^{*}$ & $57.10 \pm 9.49$ & $43.20 \pm 6.19$ \\
\hline BMl & $24.38 \pm 3.59$ & $24.88 \pm 5.71$ & $24.98 \pm 5.20$ & $23.92 \pm 3.52$ \\
\hline $\mathrm{BMC}$ & $2.66 \pm 0.41^{*}$ & $2.09 \pm 0.24^{*}$ & $3.04 \pm 0.58$ & $2.42 \pm 0.32$ \\
\hline$A C$ & $33.01 \pm 9.83$ & $29.76 \pm 4.61$ & $32.85 \pm 7.00$ & $30.17 \pm 2.92$ \\
\hline AMC & $27.99 \pm 9.75$ & $23.06 \pm 2.67$ & $27.82 \pm 5.44$ & $24.09 \pm 1.97$ \\
\hline WC & $83.34 \pm 10.90$ & $90.44 \pm 21.30$ & $86.33 \pm 10.55$ & $84.37 \pm 9.70$ \\
\hline VFA & $75.74 \pm 47.44$ & $142.21 \pm 82.66^{*}$ & $75.54 \pm 38.10$ & $95.36 \pm 50.33$ \\
\hline SBP & $136.28 \pm 18.71$ & $137.00 \pm 21.4$ & $136.04 \pm 21.62$ & $132.66 \pm 38.17$ \\
\hline DBP & $79.40 \pm 10.32^{*}$ & $75.00 \pm 7.50^{*}$ & $78.28 \pm 12.42$ & $74.45 \pm 21.24$ \\
\hline ALB & $39.95 \pm 5.81^{*}$ & $35.51 \pm 7.55^{*}$ & $41.59 \pm 4.02$ & $41.74 \pm 3.79$ \\
\hline $\mathrm{Cr}$ & $94.15 \pm 27.99^{*}$ & $45.82 \pm 29.07^{*}$ & $89.40 \pm 24.05$ & $24.05 \pm 43.53$ \\
\hline Glu & $5.67 \pm 1.43$ & $5.04 \pm 1.17$ & $5.71 \pm 1.92$ & $5.68 \pm 2.21$ \\
\hline TC & $4.45 \pm 1.15$ & $4.02 \pm 1.19$ & $4.40 \pm 1.21$ & $1.1 \pm 0.19$ \\
\hline TG & $1.14 \pm 0.51$ & $1.13 \pm 0.51$ & $1.32 \pm 0.67$ & $1.72 \pm 1.22$ \\
\hline Lym & $1.79 \pm 0.68$ & $1.62 \pm 0.59$ & $2.04 \pm 0.69$ & $1.88 \pm 0.65$ \\
\hline $\mathrm{Hb}$ & $135.83 \pm 22.02$ & $118.33 \pm 26.37^{*}$ & $140.06 \pm 16.16$ & $131.74 \pm 13.57$ \\
\hline Hyper (\%) & 15 (37.5.0) & 15 (38.4) & $76(39.0)$ & $70(42.1)$ \\
\hline Cardiac (\%) & $19(48.0)^{*}$ & $25(66.7)^{*}$ & $47(24.3)$ & $43(26.3)$ \\
\hline NC (\%) & $5(12.5)$ & $4(10.2)$ & $33(17.0)$ & $16(10.5)$ \\
\hline DM (\%) & $10(25.0)$ & $9(23.1)$ & $41(21.1)$ & $35(21.0)$ \\
\hline Smoke (\%) & $5(12.0)^{*}$ & $2(4.0)^{*}$ & $19(9.7)$ & $0(0)$ \\
\hline Drink (\%) & $11(28.0)$ & $0(0)$ & $48(25.2)$ & $0(0)$ \\
\hline Sport (\%) & $4(10.0)^{*}$ & $5(12.8)^{*}$ & $34(17.4)$ & $58(34.7)$ \\
\hline
\end{tabular}

$* P<0.05$, there were significant differences between the groups

in the Sarcopenia group were higher than the control group; the comparison between groups showed significant difference $(P<0.05)$. Correlation analysis of TNF- $\alpha$, IL- 6 levels and body composition, biochemical index is shown in Table 5. BMI was significant positively correlated with TNF- $\alpha$, and ALB was significant negatively correlated with TNF- $\alpha$ $(P<0.05)$. BMI and VFA were significant positively correlated with IL-6, whereas SMM, Hb, HDL-C, and $\mathrm{HG}$ were significant negatively correlated with IL-6 $(P<0.05)$.

(5) Multivariate linear regression analysis: With TNF- $\alpha$ and IL-6, respectively, as the dependent variables, with BMI, SMM, VFA, ALB, HDL-C, LDL-C, and $\mathrm{Hb}$ as independent variables in the Logistic multivariate regression model, the results are shown in Table 6. It suggested that ALB and BMI had effective predictive value for TNF- $\alpha(P<0.05)$, and VFA was the independent risk factor for the development of IL-6 $(P<0.05)$.

\section{Discussion}

Muscle reduction syndrome was defined as sarcopenia for the first time by Rosenberg in 1989 in the consensus published by EWGSOP in 2010 and AWGS in 2015. Since then, more attention has been given to sarcopenia by 
Table 2 Correlation analysis of Spearman with body composition and muscle decrease

\begin{tabular}{lrl}
\hline & \multicolumn{1}{l}{$r$} & $\boldsymbol{P}$ \\
\hline ICW & 0.760 & $0.000^{*}$ \\
ECW & 0.811 & $0.000^{*}$ \\
Pro & 0.762 & $0.000^{*}$ \\
FAT & -0.307 & $0.040^{*}$ \\
FFM & 0.780 & $0.000^{*}$ \\
BMI & 0.306 & 0.078 \\
BMC & 0.674 & $0.000^{*}$ \\
AC & 0.483 & $0.004^{*}$ \\
AMC & 0.578 & $0.000^{*}$ \\
WC & 0.160 & 0.366 \\
VFA & -0.242 & 0.167 \\
\hline
\end{tabular}

${ }^{*} P<0.05$, there was a significant difference

Table 3 Correlation analysis between Spearman and biochemical indexes of muscular disorders

\begin{tabular}{lll}
\hline & $\boldsymbol{r}$ & $\boldsymbol{P}$ \\
\hline SBP & 0.071 & 0.692 \\
DBP & 0.343 & $0.047^{*}$ \\
ALB & 0.367 & $0.035^{*}$ \\
Cr & 0.420 & $0.015^{*}$ \\
Glu & 0.202 & 0.259 \\
TC & 0.223 & 0.211 \\
TG & 0.162 & 0.367 \\
Lym & 0.291 & 0.101 \\
Hb & 0.274 & 0.123 \\
\hline
\end{tabular}

$* P<0.05$, there was a significant difference

global medical communities; and strategies for diagnosis and treatment have been gradually improved. Sarcopenia is a prevalent disease in the elderly. The results of latest studies have revealed that the morbidity rate of sarcopenia was $5-13 \%$ in people aged between 60 and 70 years, and that this rate was $11-50 \%$ in populations of over 80 years $[5,6]$. Since this can increase falling down and fracture risks in the elderly, and is closely related to the increase in disability and mortality rates [7-9], it is very necessary to explore the characteristics and pathogenesis of this disease to reduce its occurrence and development, so as to improve the life quality of the elderly and reduce the re-admission rate and medical cost. The reason why the elderly are prone to suffer from sarcopenia may be related to multiple factors $[1,10]$, such as abnormal protein metabolism, oxidative stress, and chronic inflammations. Based on the findings of this study, specific analyses are as follows.

\section{Risk factors of sarcopenia}

Studies have shown that smoking and poor exercise habits were related to sarcopenia, which was consistent with previous research results. A large-scale study on sarcopenia in the elderly in Hong Kong indicated that smoking is one of the risk factors of sarcopenia. It was also found that quitting smoking could increase the amount of muscle content, fat content, and weight. Therefore, smoking cessation may be one of the means to prevent and treat sarcopenia. Exercise is also an effective means to prevent and treat sarcopenia, which can change the quality and strength of skeletal muscles, improve balance ability, and play a good role in the prevention and treatment of sarcopenia in the elderly. Furthermore, resistive exercise can increase the cross-sectional area and number of muscles through the reduction of body fat, especially subcutaneous and abdominal fats [11], which result in increased muscle mass. Li et al. found that resistance training can effectively increase the muscle content of limbs and reduce the level of inflammatory factors [12]. The serum level of IL- 6 may be decreased by $14.4 \%$ in an elderly who can conduct regular exercises for 24 weeks. Therefore, exercise can reduce the level of serum IL- 6 and inflammatory reaction to reduce the incidence of sarcopenia.

Relationship between inflammatory factors and sarcopenia Our research results indicated that IL- 6 and TNF- $\alpha$ serum levels in elderly subjects with sarcopenia were higher than levels in the control group, and BMI VFA were independent risk factors for inflammatory cytokines IL- 6 by multiple regression analysis. These were consistent with the results of a previous similar study [13]. As age increased, a chronic low-level systemic inflammatory reaction occurred in body; and inflammation is a concomitant reaction of cell senescence and organism aging. It was found in the study that, accompanied with organism aging, inflammatory factors in vivo increased significantly, and serum proinflammatory factors such as TNF- $\alpha$, IL-6, and C-reactive protein (CRP) could increase

Table 4 Comparison of levels of inflammatory cytokines

\begin{tabular}{|c|c|c|c|}
\hline Inflammatory factors & Muscle decrease disease group & Non-muscle decrease disease group & $P$ \\
\hline TNF-a $(p g / m l)$ & $165.39 \pm 19.49$ & $148.79 \pm 26.06$ & 0.01 \\
\hline IL-6 (pg/ml) & $49.77 \pm 22.14$ & $39.72 \pm 29.53$ & 0.03 \\
\hline
\end{tabular}


Table 5 Relationship between inflammatory factors and body composition and biochemical indexes

\begin{tabular}{|c|c|c|c|c|}
\hline \multirow[t]{2}{*}{ Variable } & \multicolumn{2}{|l|}{ TNF-a } & \multicolumn{2}{|l|}{ IL-6 } \\
\hline & $r$ & $P$ & $r$ & $P$ \\
\hline ALB & -0.266 & $0.024^{*}$ & -0.044 & 0.373 \\
\hline SMM & 0.184 & 0.087 & -0.238 & $0.039^{*}$ \\
\hline HDL-C & -0.099 & 0.233 & -0.389 & $0.002^{* *}$ \\
\hline $\mathrm{Hb}$ & 0.034 & 0.400 & -0.304 & $0.011^{*}$ \\
\hline BMI & 0.346 & $0.008^{* *}$ & 0.373 & $0.004^{* *}$ \\
\hline VFA & 0.212 & 0.059 & 0.464 & $0.000^{* *}$ \\
\hline LDL-C & 0.162 & 0.117 & 0.020 & 0.440 \\
\hline$H G$ & 0.411 & 0.070 & -0.450 & $0.048^{*}$ \\
\hline
\end{tabular}

${ }^{*} P<0.05$, there was a significant difference

2-4 times, even in elderly without chronic diseases. Visceral fat tissue (VFA) is known to be distributed predominantly in the abdominal cavity. Recently, evidence supports that visceral fat tissue contains large number of adipocytes and is more hormonally active since there are more glucocorticoid and androgen receptors in comparison with subcutaneous fat [14]. Another evidence suggests that as adipose tissue increases the amount of the anti-inflammatory cytokine decreases, whereas there is an increase in the level of the proinflammatory molecules such as leptin, TNF- $\alpha$, IL-1, and IL-6 [15]. In the elderly, increased serum levels of inflammatory factors were closely related to the reduction of skeletal muscle content and strength induced by senescence $[16,17]$. Moreover, increased serum IL-6 levels are positively associated with disability rate and mortality [18], and negatively correlated to the recoveries of walking and muscle function in the fracture healing process in elderly women [19]. These large-scale study results revealed that supersensitive CRP and IL- 6 are important predictors of muscle strength reduction within the next 3 years of middle- and old-aged people over 55 years [20]. Therefore, inflammatory reaction plays an important role in the development of elderly sarcopenia.

At the same time, our study found that ALB was an effective predictor of inflammatory factor TNF- $\alpha$. Sullivan's study found that inflammation appears to be a more powerful determinant of albumin, particularly CRP, and IL-6, and its change during the hospitalization than is nutrient intake in elderly patients admitted to a TCU [21]. Another study found, TNF- $\alpha$, IL-10, and markers of oxidative stress were in negative correlation with HDL cholesterol and albumin. Resolution of the inflammation is probably the primary prerequisite to the normalization of the serum albumin [22].

According to the findings in the study, the possible mechanisms of IL- 6 in the induction of sarcopenia are as follows. (1) Inflammatory factors can inhibit the synthesis of muscle proteins, accelerate protein decomposition, and upregulate the expression of muscle growth inhibitory factor myostatin and muscle atrophy proteins, F-box-1 Atrogin-1, etc., so as to accelerate protein catabolism and promote skeletal muscle consumption [23, 24]. Muscle tissues contain the most amounts of proteins in the human body, and IL-6 can lead to the reduction of muscle mass by means of disrupting protein synthesis and directly participating in protein decomposition. Furthermore, high levels of IL- 6 can inhibit the anabolism of muscle tissue in IGF-I [25, 26]. (2) Age-related changes in body composition can easily cause increased cytokine products. As an organism ages, adipose cells increases, and IL- 6 and TNF- $\alpha$ can easily be secreted, which aggravate inflammatory reactions. Furthermore, this would lead to the reduction in muscle mass and strength, resulting in the occurrence of sarcopenia $[1,10]$. (3) Insulin resistance is another mechanism of sarcopenia. Insulin not only has the function of reducing blood sugar, but also accelerates the protein synthesis of targets on which

Table 6 Logistic regression analysis of inflammatory cytokines and other indexes

\begin{tabular}{|c|c|c|c|c|c|c|c|c|}
\hline & \multicolumn{3}{|l|}{ TNF- $a$} & \multirow[t]{2}{*}{$P$} & \multicolumn{3}{|l|}{ IL-6 } & \multirow[t]{2}{*}{$P$} \\
\hline & $\beta$ & Std & $t$ & & $\beta$ & Std & $t$ & \\
\hline Constant & -268.119 & 145.975 & -1.837 & 0.072 & -9.738 & 93.284 & -2.347 & 0.021 \\
\hline ALB & -7.763 & 3.045 & -2.550 & $0.014^{*}$ & 1.638 & 1.946 & 0.842 & 0.404 \\
\hline BMI & 9.192 & 3.036 & 3.027 & $0.004^{*}$ & 1.183 & 1.940 & 0.609 & 0.545 \\
\hline SMM & -0.522 & 1.255 & -0.416 & 0.679 & -0.325 & 0.802 & -0.405 & 0.687 \\
\hline LDL & 5.160 & 7.027 & 0.734 & 0.466 & 0.280 & 4.491 & 0.062 & 0.951 \\
\hline $\mathrm{HDL}-\mathrm{C}$ & 14.285 & 21.176 & 0.675 & 0.503 & 17.458 & 13.532 & 1.290 & 0.203 \\
\hline $\mathrm{Hb}$ & -0.846 & 0.511 & -1.655 & 0.105 & -0.129 & 0.327 & -0.395 & 0.695 \\
\hline VFA & 0.357 & 0.516 & 0.692 & 0.492 & 0.644 & 0.187 & 3.445 & $0.001^{*}$ \\
\hline
\end{tabular}

* $P<0.05$, there was a significant difference 
the muscle fibers act. At the same time, the uptake of calcium in cells is affected by insulin. Insulin resistance may lead to decreased calcium uptake, which is not conducive to muscle contraction [27]. Therefore, elevated IL- 6 and TNF- $\alpha$ serum levels can lead to insulin resistance and the occurrence of sarcopenia. (4) Recent studies have indicated that inflammatory reactions may also lead to the occurrence of sarcopenia through triggering mitochondrial abnormalities in the manner of disturbing the number or origin of mitochondria [28]. A study on the correlation between TNF- $\alpha$ and sarcopenia revealed that elevated levels of TNF- $\alpha$ are associated with the decline in muscle mass and strength. In addition, a study on 5-year continuous observations on elderly subjects aged within 70-79 years and 4-year continuous observations on elderly subjects aged $>85$ years has indicated that plasma TNF- $\alpha$ levels can predict the decline in muscle strength; wherein, as every standard deviation of TNF- $\alpha$ level increased, 1.2-1.3 kg of HG strength would be decreased [29].

Inflammatory reaction is just one of the factors for the occurrence of sarcopenia. Other factors include nutrition issues and hormone level decline. Sarcopenia would become one of the main reasons of disability and reduction in the quality of life in the elderly. In recent years, although many scholars have explored this in various aspects, its pathological mechanism remains unclear and there are no standard diagnostic criteria. In order to determine the effective method for preventing and treating this disease, it is necessary to proceed with more large-scale clinical studies.

\section{Conclusions}

The occurrence of elderly sarcopenia is related to poor exercise habits, disease history, and nutritional status. The emergence of Sarcopenia was accompanied by increased levels of inflammation factors TNF- $\alpha$ and IL- 6 . Plasma albumin, BMI, and VFA were inflammatory factor predictors of TNF and IL-6. Prohibiting smoking habits and exercise could reduce the incidence of sarcopenia.

\begin{abstract}
Abbreviations
HG: handgrip strength; FFM: fat-free mass; BMC: bone mineral content; ALB: plasma albumin; CrL: serum creatinine; FAT: body fat content; IL-6: inflammatory factors interleukin-6; TNF-a: tumor necrosis factor-a; ASMI: appendicular skeletal muscle index; SD: standard deviations; BIA: bioelectrical impedance analysis; ICW: intracellular water; ECW: extracellular water; SLM: appendicular skeletal lean mass; SMM: skeletal muscle mass; FAT: fat content; FFM: fat-free body mass; BMC: bony mineral content; Pro: protein content; BCM: body cell mass; BMI: body mass index; AC: arm circumference; AMC: arm muscle circumference; WC: waist circumference; Hb: hemoglobin; Lym: lymphocyte; TC: total cholesterol; TG: triacylglycerol; Glu: glucose; ALB: serum albumin; Cr: creatinine.
\end{abstract}

\section{Authors' contributions}

A-LB has made substantial contributions to conception and design; $\mathrm{H}-\mathrm{YH}$ and YDR have been involved in acquisition of data, analysis, and interpretation of data; JW, J-XW and X-ZZ have been involved in drafting the manuscript and revising it critically for important intellectual content; A-LB has given final approval of the version to be published. All authors read and approved the final manuscript.

\section{Competing interests}

The authors declare that they have no competing interests.

\section{Availability of data and materials}

These data will not be shared, because in recent years, although many scholars have explored this in various aspects, its pathological mechanism remains unclear and there are no standard diagnostic criteria. In order to determine the effective method for preventing and treating this disease, it is necessary to proceed with more large-scale clinical studies.

\section{Consent for publication}

All written informed consent was obtained from all participants.

\section{Ethics and consent statement}

This study was conducted in accordance with the Declaration of Helsinki. This study was conducted with approval from the Ethics Committee of our hospital.

\section{Publisher's Note}

Springer Nature remains neutral with regard to jurisdictional claims in published maps and institutional affiliations.

Received: 25 January 2017 Accepted: 30 June 2017

Published online: 12 July 2017

\section{References}

1. Walrand S, Guillet C, Salles J, Cano N, Boirie Y. Physiopathological mechanism of sarcopenia. Clin Geriatr Med. 2011;27:365-85.

2. Visser M, Pahor M, Taaffe DR, Goodpaster BH, Simonsick EM, Newman AB, Nevitt M, Harris TB. Relationship of interleukin- 6 and tumour necrosis factor-alpha with muscle mass and muscle strength in elderly men and women: the Health ABC Study. J Gerontol A Biol Sci Med Sci. 2002;57:M326-32.

3. Cesari M, Kritchevsky SB, Baumgartner RN, Atkinson HH, Penninx BW, Lenchik L, Palla SL, Ambrosius WT, Tracy RP, Pahor M. Sarcopenia, obesity, and inflammation:results from the trial of angiotensin converting enzyme inhibition and novel cardiovascular risk factors study. Am J Clin Nutr. 2005:82:428-34.

4. Schrager MA, Metter EJ, Simonsick E, Ble A, Bandinelli S, Lauretani F, Ferrucci L. Sarcopenic obesity and inflammation in the InCHIANTI study. J Appl Physiol. 2007;102:919-25.

5. Von Haehling S, Morley JE, Anker SD. An overview of sarcopenia:facts and numbers on prevalence and clinical impact. J Cachexia Sarcopenia Muscle. 2010;1:129-33.

6. Morley JE. Sarcopenia in the elderly. Fam Pract. 2012;29(Suppl 1):i44-8.

7. Cruz-Jentoft AJ, Baeyens JP, Bauer JM, Boirie Y, Cederholm T, Landi F, Martin FC, Michel JP, Rolland Y, Schneider SM, Topinková E, Vandewoude M, Zamboni M, European Working Group on Sarcopenia in Older People. Sarcopenia: European consensus on definition and diagnosis:report of the European Working Group on Sarcopenia in older people. Age Ageing. 2010;39:412-23.

8. Woo N, Kim SH. Sarcopenia influences fall-related injuries in community—dwelling older adults. Geriatr Nurs. 2014;35:279-82.

9. Arango-Lopera VE, Arroyo P, Gutiérrez-Robledo LM, Pérez-Zepeda MU, Cesari M. Mortality as an adverse outcome of sarcopenia. J Nutr Health Aging. 2013;17:259-62.

10. Newman AB, Kupelian V, Visser M, Simonsick E, Goodpaster B, Nevitt M, Kritchevsky SB, Tylavsky FA, Rubin SM, Harris TB, Health ABC Study Investigators. Sarcopenia: alternative definitions and association with lower extremity function. J Am Geriatr Soc. 2003;51:1602-9. 
11. Woods JA, Wilund KR, Martin SA, Kistler BM. Exercise, inflammation and aging. Aging Dis. 2012;3(1):130-40.

12. Shugang Li, Yuan Zhou. Effects of resistance training on elderly muscle content and serum levels of inflammatory factors. China J Gerontol. 2014:23:6659-61.

13. Stenholm S, Rantanen T, Heliovaara M, Koskinen S. The mediating role of C-reactive protein and handgrip strength between obesity and walking limitation. J Am Geriatr Soc. 2008;56(3):462-9.

14. Ibrahim MM. Subcutaneous and visceral adipose tissue: structural and functional differences. Obes Rev. 2010;11:11-8.

15. Stefanyk LE, Dyck DJ. The interaction between adipokines, diet and exercise on muscle insulin sensitivity. Curr Opin Clin Nutr Metab Care. 2010;13:255-9.

16. Taaffe DR, Harris TB, Ferrucci L, Rowe J, Seeman TE. Cross-sectional and prospective relationships of interleukin-6 and C-reactive protein with physical performance in elderly persons: MacArthur studies of successful aging. J Gerontol A Biol Sci Med Sci. 2000;55:M709-15.

17. Visser M, Pluijm SM, Stel VS, Bosscher RJ, Deeg DJ, Longitudinal Aging Study Amsterdam. Physical activity as a determinant change in mobility performance: the longitudinal aging study Amsterdam. J Am Geriatr Soc. 2002;50(11):1774-81.

18. Schrager MA, Metter EJ, Simonsick E, Ble A, Bandinelli S, Lauretani F, Ferrucci L. Sarcopenic obesity and inflammation in the InCHIANTI study. J App Physiol. 2007;102(3):919-25.

19. Visser M, Pahor M, Taaffe DR, Goodpaster BH, Simonsick EM, Newman AB, Nevitt M, Harris TB. Relationship of interleukin-6 and tumor necrosis factor-alpha with muscle mass and muscle strength in elderly men and women: the Health ABC Study. J Gerontol A Biol Sci Med Sci. 2002:57:326-32.

20. Miller RR, Shardell MD, Hicks GE, Cappola AR, Hawkes WG, Yu-Yahiro JA, Magaziner J. Association between interleukin-6 and lower extremity function after hip fracture-the role of muscle mass and strength. J Am Geriatr Soc. 2008;56(6):1050-6.
21. Sullivan $D H$, Johnson LE, Dennis RA, et al. Interrelationships among albumin, nutrient intake, and inflammation in elderly recuperative care patients. J Nutr Health Aging. 2011;15(4):311-5.

22. Vavrova L, Rychlikova J, Mrackova M, et al. Increased inflammatory markers with altered antioxidant status persist after clinical recovery from severe sepsis: a correlation with low HDL cholesterol and albumin. Clin Exp Med. 2016;16(4):557-69.

23. Schaap LA, Pluijm SM, Deeg DJ, Visser M. Inflammatory markers and loss of muscle mass (sarcopenia) and strength. Am J Med. 2006;119(526):e517-29.

24. Ikizler TA. Nutrition, inflammation and chronic kidney disease. Curr Opin Nephrol Hypertens. 2008;17:162-7.

25. Cheung WW, Paik KH, Mak RH. Inflammation and cachexia in chronic kidney disease. Pediatr Nephrol. 2010;25:711-24.

26. Bartlett DB, Firth CM, Phillips AC, Moss P, Baylis D, Syddall H, Sayer AA, Cooper C, Lord JM. The age-related increase in lowgrade systemic inflammation (Inflammaging) is not driven by cytomegalovirus infection. Aging Cell. 2012:11(5):912-5.

27. Serri O, St-Jacques P, Sartippour M, Renier G. Alterations of monocyte function in patients with growth hormone $(\mathrm{GH})$ deficiency: effect of substitutive GH therapy. J Clin Endocrinol Metab. 1999;84:58-63.

28. Rajhans R, Nair HB, Nair SS, Cortez V, Ikuko K, Kirma NB, Zhou D, Holden AE, Brann DW, Chen S, Tekmal RR, Vadlamudi RK. Modulation of in situ estrogen synthesis by proline-, glutamic acid-, and leucine-rich protein-1: potential estrogen receptor autocrine signaling loop in breast cancer cells. Mol Endocrinol. 2008;22(3):649-64.

29. Marzetti E, Calvani R, Cesari M, Buford TW, Lorenzi M, Behnke BJ, Leeuwenburgh $C$. Mitochondrial dysfunction and sarcopenia of aging: from signaling pathways to clinical trials. Int J Biochem Cell Biol. 2013;45:2288-301.

\section{Submit your next manuscript to BioMed Central and we will help you at every step:}

- We accept pre-submission inquiries

- Our selector tool helps you to find the most relevant journal

- We provide round the clock customer support

- Convenient online submission

- Thorough peer review

- Inclusion in PubMed and all major indexing services

- Maximum visibility for your research

Submit your manuscript at www.biomedcentral.com/submit
() Biomed Central 